\title{
Low-Cost Electrical Beam Scanning Leaky-Wave Antenna Based on Bent Corrugated Substrate Integrated Waveguide
}

\author{
Tian Lou, Xue-Xia Yang, Senior Member, IEEE, Houtong Qiu, Qi Luo, Steven Gao, Senior Member, \\ IEEE
}

\begin{abstract}
This letter presents a novel low-cost leaky-wave antenna (LWA) with the fixed-frequency beam-scanning capability. An improved half-mode CSIW (HM-CSIW) structure is proposed as the guiding wave structure to reduce the transverse size of the antenna. A novel electronic phase-shifting structure, composed of fan-shaped open stubs with different sizes and PIN diodes, is proposed for beam scanning. This LWA uses interdigital slots as radiating elements, and the phase-shifting structure is placed between the adjacent radiating elements. By changing the switching states of these PIN diodes, the phase difference between the adjacent radiating elements can be controlled. To verify the concept, one prototype of the $2 \times 6$ array antenna at $C$ band is designed, simulated, fabricated and measured. The antenna demonstrates a beam-scanning range of $25^{\circ}\left(34^{\circ}-59^{\circ}\right)$ at fixed frequency, a peak gain of $12.4 \mathrm{dBi}$ with the gain variation less than $2.3 \mathrm{~dB}$. The antenna has low cost and can be easily fabricated using standard Printed Circuit Board (PCB) process.
\end{abstract}

Index Terms-Leaky-wave antenna, beam scanning antenna, substrate integrated waveguide, PIN diode.

\section{INTRODUCTION}

$\mathrm{W}$ ITH the advantages of saving power, high security and decreasing interferences, beam scanning antennas are required in many civilian and military applications $[1,2]$. Since the first known microstrip planar leaky-wave antenna (LWA) invented in the late $1970 \mathrm{~s}$, the planar LWA has attracted increasing attention because of its simple feeding network, low profile, and frequency-scanning capability. Recently, based on LWA, different types of frequency-scanning antenna were proposed, such as microstrip LWAs [3, 4], substrate integrated waveguide (SIW) LWAs [5, 6] and parallel plate waveguide LWA [7]. However, the frequency-scanning antennas need a wide operation band, which is not suitable for most wireless

Manuscript received ****, 2018. This work was supported by the National Natural Science Foundations of China (61771300).

X. X. Yang is with the Key Laboratory of Specialty Fiber Optics and Optica Access Networks, Shanghai Institute for Advanced Communication and Data Science, Shanghai University, Shanghai 200444, China (phone: 0086-21-66136292; email: yang.xx@shu.edu.cn).

T. Lou and H. Qiu are with the School of Communication and Information Engineering, Shanghai University, Shanghai 200072, China (email: loutian@shu.edu.cn; 810970463@qq.com).

S. Gao and Q. Luo are with the School of Engineering and Digital Arts, University of Kent, UK (email: S.Gao@kent.ac.uk; Q.Luo@kent.ac.uk). communication systems.

By loading binary switches and metal shorted patches on the half-width microstrip line, a LWA with beam scanning operating at a fixed frequency in C-band was demonstrated in [8]. A beam scanning LWA operating at $2.4 \mathrm{GHz}$ was proposed in [9] by using metamaterial-based phase shifters. Another solution to achieve beam scanning at a fixed frequency is loading varactors between the radiation elements [10]. The SIW technology brings the benefits of changing the traditional rectangular waveguide to the planar microwave integrated circuit technology [11]. Due to its well-known features of being light weight, low profile, low cost and ease of fabrication, the SIW is suitable for designing the LWA [12-14]. However, the top and bottom conductors of the SIW is not DC-isolated because they are connected by the metallic vias. So the SIW is not convenient for DC bias and integration of active devices. Chen and Eccleston proposed the corrugated SIW (CSIW), which uses open-circuit quarter-wavelength microstrip stubs in place of metallic vias to artificially create the electric side-walls and maintain the DC-isolation between the top and bottom conductors [15]. This structure can support the same $\mathrm{TE}_{10}$ mode as the SIW and is easy to integrate with active components [16]. However, CSIW has a wide transverse dimension, which is not suitable for the array design. In order to reduce the transverse size of the CSIW, an improved CSIW structure, denoted the bent CSIW (BCSIW), is proposed in this letter. By using a bent open-circuit quarter-wavelength microstrip stub, the size of the CSIW is reduced by about $17 \%$.

In this letter, based on the proposed BCSIW structure, a LWA with fixed-frequency beam scanning capability is designed. This LWA uses interdigital slots as the radiating elements, and scans its main beam at a fixed frequency by placing phase-shifting structures between adjacent radiating elements. In order to combine the phase-shifting structure with the LWA, half-mode BCSIW (HM-BCSIW) is used in this design. A novel electronic phase-shifting structure, which mainly consists of 4 fan-shaped open-circuit microstrip stubs of different sizes and 6 PIN diodes, is connected to the free edge of the HM-BCSIW. The beam scanning is achieved by changing the DC bias voltages of the 6 PIN diodes on each phase-shifting structure. A prototype antenna was simulated, fabricated and measured to validate the proposed design. Because the top and bottom conductors of this antenna are 
DC-isolated, it can be easily combined with other active components and devices.

\section{ANTENNA DESIGN AND ANALYSIS}

\section{A. HM-BCSIW Guiding Wave Structure Evolution}

Fig. 1 shows the evolution of the proposed HM-BCSIW. The CSIW uses open-circuit quarter-wavelength microstrip stubs (Fig. 1 (b)) in place of the metallic vias in the conventional SIW (Fig. 1 (a)) to artificially create the electric side-walls and maintain the DC-isolation between the top and bottom conductors [15]. By using a bent open-circuit quarter-wavelength microstrip stub (Fig.1 (c)), the CSIW transverse size is reduced, which is denoted as BCSIW. In order to further reduce the size of the CSIW and connect to the phase-shifting structures in the subsequent antenna design, the half-mode BCSIW (HM-BCSIW) structure (Fig.1 (d)) is suggested.

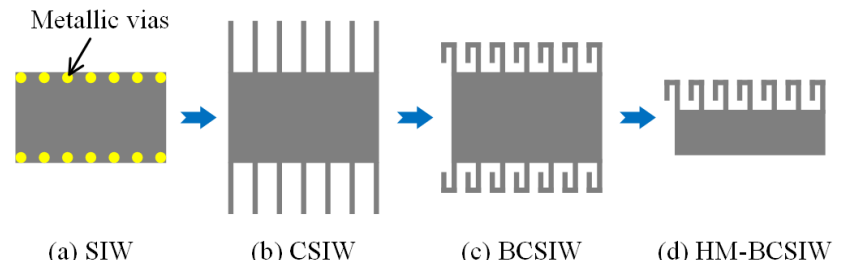
(a) SIW
(b) CSIW
(c) BCSIW
(d) HM-BCSIW

Fig. 1. Evolution of the HM-BCSIW.

Fig. 2 shows the electrical field distributions of the four SIW structures at $5.8 \mathrm{GHz}$. Those of the CSIW and BCSIW is similar to that of the traditional SIW, which show they operate on the same mode of $\mathrm{TE}_{10}$. Correspondingly, the operation mode for HM-BCSIW is the $\mathrm{TE}_{0.5,1}$ mode. Both the bent open-circuit quarter-wavelength microstrip stub and the straight one form the artificial electric side-walls.

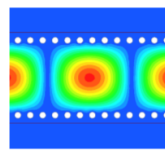

(a)

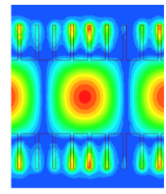

(b)

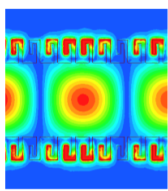

(c)

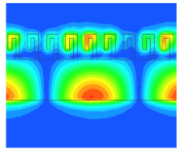

(d)
Fig. 2. The electrical field distributions of (a) SIW (b) CSIW (c) BCSIW (d) $\mathrm{HM}-\mathrm{BCSIW}$ at $5.8 \mathrm{GHz}$.

\section{B. HM-BCSIW Beam Scanning LWA Design}

Fig. 3 shows the configuration of the proposed electrical beam scanning LWA. The substrate used for this design is F4B with the relative permittivity of 2.2 and the loss tangent of 0.001 . The substrate thickness is $1.5 \mathrm{~mm}$. The antenna composed of the HM-BCSIW leaky-wave antenna, the electronic phase-shifting structure and the microstrip to HM-BCSIW transition structure.

The LWA uses HM-BCSIW as the guiding wave structure. Referring to the SIW based LWA design in [12], by etching interdigital slots on the top conductor of the HM-BCSIW as the radiating elements, a HM-BCSIW leaky-wave antenna is developed. Two HM-BCSIW leaky-wave antennas are placed symmetrically for increasing the antenna gain. An electronic phase-shifting structure is placed between adjacent radiating elements for the fixed-frequency beam scanning capability of the LWA. By electrically changing the phase difference between adjacent radiating elements, the beam scanning is achieved. There are $2 \times 6$ radiating elements and $2 \times 5$ phase-shifting structures in the prototyped antenna.

The microstrip to HM-BCSIW transition structure, which includes a $50 \Omega$ microstrip line, a microstrip power divider and a tapered microstrip line, is used as feed structure of the LWA. The tapered microstrip line is used to excite the $\mathrm{TE}_{10}$ mode of the HM-BCSIW. By optimizing the widths $W_{4}, W_{5}$ and the length $L_{4}$ of the tapered microstrip line, the structure can achieve a good impedance matching. The antenna is fed from one end, and the other end is terminated with a $50 \Omega$ coaxial load to suppress the reflected waves.

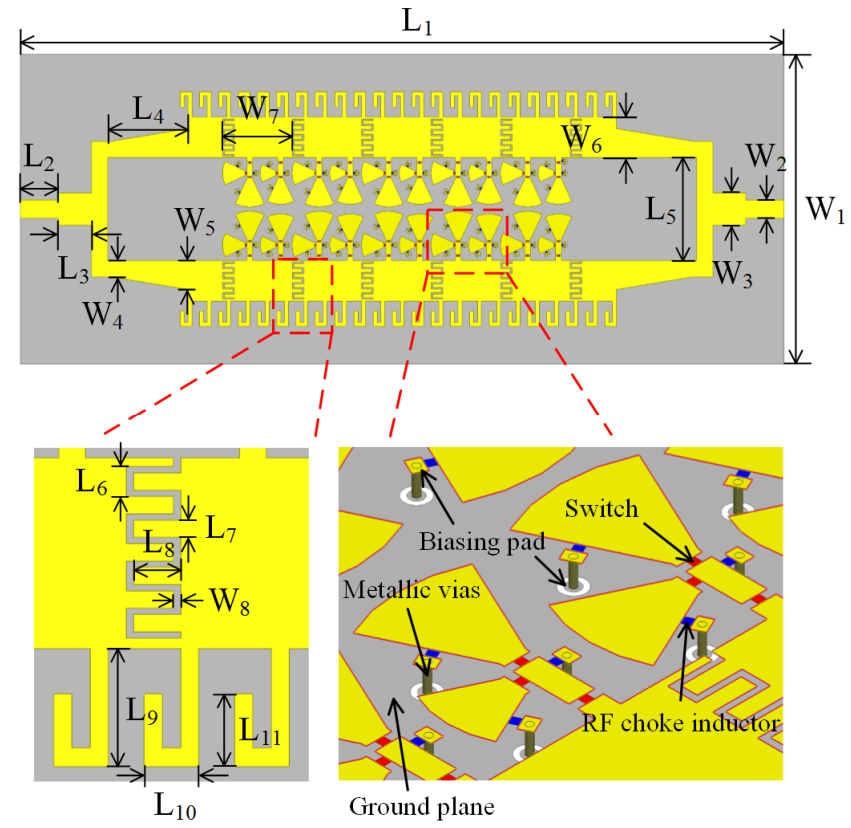

Fig. 3. Configuration of the proposed electrical beam scanning LWA.

The detailed dimensions of the proposed electronic phase-shifting structure are shown in Fig. 4. The phase-shifting structure is composed of 2 phase shifting units, while each phase shifting unit consists of 2 fan-shaped open stubs with different sizes. In order to make the structure compact, the 2 fan-shaped open stubs are positioned perpendicularly to each other and are connected to a rectangular patch of $1.5 \mathrm{~mm} \times 3.25 \mathrm{~mm}$ in size by binary switches, respectively. The patch is connected to the free edge of the HM-BCSIW by a binary switch and a microstrip line of $1.5 \mathrm{~mm} \times 1 \mathrm{~mm}$ in size. The dimensions of the 4 fan-shaped open stubs used in the electronic phase-shifting structure are $R_{1}, R_{2}, R_{3}$ and $R_{4}$, respectively, and the binary switches are numbered as $S 1 \sim S 6$ whose positions are shown in Fig. 4. The PIN diode is used as the binary switch in this design. The switching state of the PIN diode is controlled by the DC bias voltages. When the diode is forward biased, the diode operates in the "on" state. When the diode is reverse biased, the diode operates in the "off" state. A 
DC biasing circuit is designed to independently control the switching state of each PIN diode. The DC biasing circuit consists of the RF choke inductors, the top biasing pads, the bottom biasing pads and the metallic vias. The bottom biasing pads are separated from the ground plane by etching annular slots. The DC bias voltages supply is on the bottom biasing pads to minimize the effect of DC circuits on the antenna RF performance. The widths of the slits for placing the PIN diode and the RF choke inductor are $W_{9}$ and $W_{10}$, respectively.

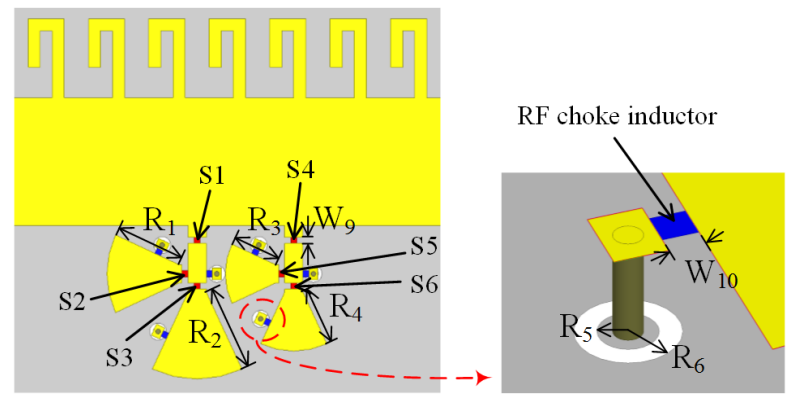

Fig. 4. Structure of the electronic phase-shifting structure.

The operation principle of the electronic phase-shifting structure is as follows. The phase-shifting structure is connected to the HM-BCSIW. By controlling the states of the binary switches $S 1 \sim S 6$, the number and size of the fan-shaped open stubs loaded on the HM-BCSIW are different. For example, when switches $S 1$ and $S 2$ are set to "on" state and switches $S 3 \sim S 6$ are set to "off" state, the loading on the HM-BCSIW is the stub with the size of $R_{1}$. When switches $S 2$ and $S 5$ is set to "off" state and the other switches are set to "on" state, the loading on the HM-BCSIW are the two stubs with the size of $R_{2}$ and $R_{4}$. The "on" and "off" states of each switch are denoted as " 1 " and " 0 ", respectively. Thus the operation states of this electronic phase-shifting structure can be described by using a 6-bit binary codes.

\section{Simulation Analysis}

The PIN diode used in the simulation and measurement are BAR50-02L from Infineon Company. The equivalent circuit model used in the simulation is shown in Fig. 5. The PIN diode can be equivalent to a forward resistance $R_{\mathrm{f}}=3 \Omega$ in the "on (1)" state, and a parallel circuit consisting of a capacitance $C_{\mathrm{p}}=0.1$ $\mathrm{pF}$ and a reverse resistance $R_{\mathrm{p}}=5 \mathrm{k} \Omega$ in the "off (0)" state. The additional packaging parasitic inductance $L_{\mathrm{s}}=0.4 \mathrm{nH}$ is also included in the simulation model.

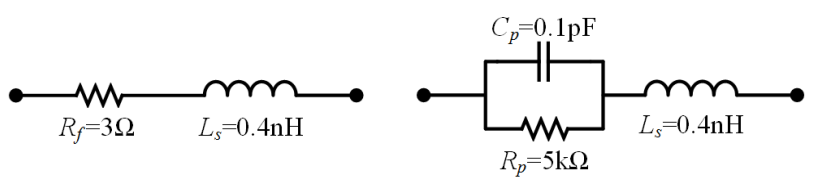

(a)

(b)

Fig. 5. Simulation models of PIN diode in (a) "on" and (b) "off" states.

To illustrate the beam scanning effect of the antenna with different values and number of fan-shaped open stubs, Fig. 6 shows the radiation pattern with different values of $R_{4}$ for the switch states of "000101" and "101101". When the size of the fan-shaped open stub $R_{4}$ varies from $3.6 \mathrm{~mm}$ to $7 \mathrm{~mm}$, the scan-angle ranges corresponding to the switch states "000101" and "101101" are $45^{\circ}-52^{\circ}$ and $39^{\circ}-46^{\circ}$, respectively.

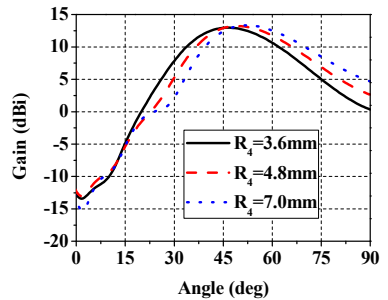

(a)

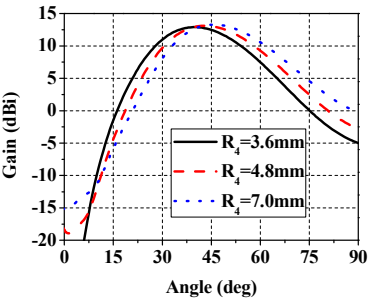

(b)
Fig. 6. The radiation pattern with different values of $R_{4}$ for (a) "000101" state and (b) "101101" state. (The sizes of $R_{1}, R_{2}$ and $R_{3}$ are unchanged with the values of $5.4 \mathrm{~mm}, 7.1 \mathrm{~mm}$ and $3.6 \mathrm{~mm}$, respectively.)

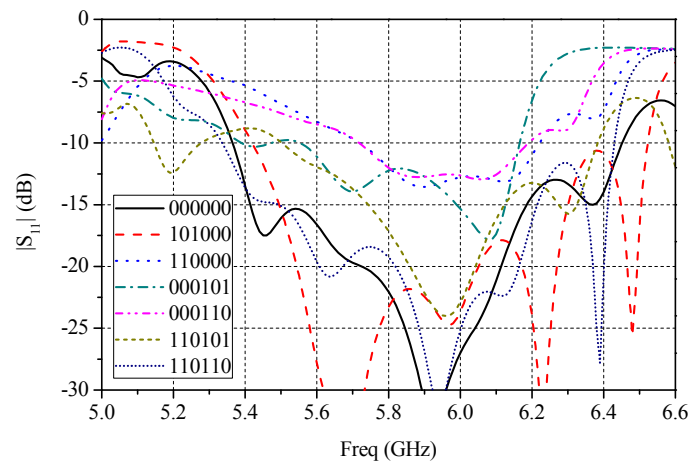

Fig. 7. Simulated reflection coefficients of the proposed antenna for different switching states.

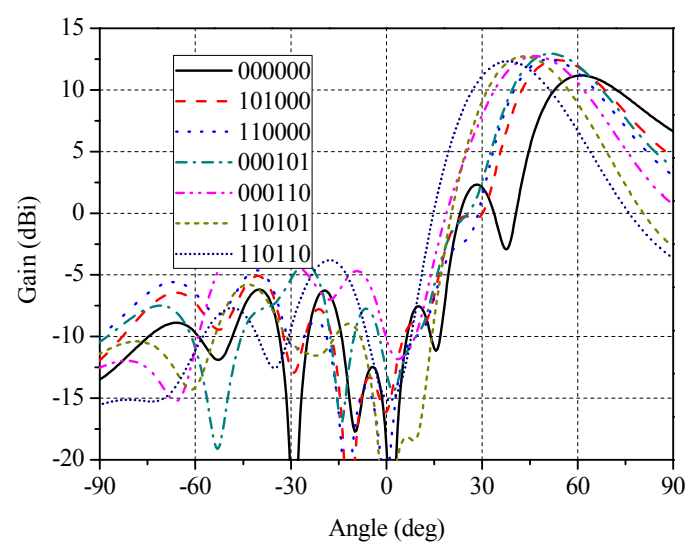

Fig. 8. Simulated radiation patterns of the proposed antenna for different switching states at $5.8 \mathrm{GHz}$.

The simulated reflection coefficients for different switching states are plotted in Fig. 7. The simulated bandwidth for these switching states of the reflection coefficient less than $-10 \mathrm{~dB}$ is $5.72-6.16 \mathrm{GHz}$. Fig. 8 shows the radiation patterns of these switching states at the operation frequency of $5.8 \mathrm{GHz}$. As can be seen, the main beam directions for some switching states are almost the same. For example, the main beam direction for switching states of "110000" and "000101" are $51^{\circ}$ and $51.5^{\circ}$, respectively, which are very close to each other. Therefore, in practice, only parts of the switching states are useful. Considering the reflection coefficient of different switching states, 5 states corresponding to different scanning angles are selected as the preset scanning states of the antenna, which are 
"000000", "101000", "000101", "110101" and "110110". The maximum simulated gain of these 5 states is $12.9 \mathrm{dBi}$. Within the scan-angle ranges from $37^{\circ}$ to $61^{\circ}$, the scanning gain variation is less than $1.8 \mathrm{~dB}$.

\section{MEASUREMENT AND DiscUSSION}

To evaluate the performance of the proposed LWA, a prototype was fabricated and measured. Table I lists the main dimensions of the antenna. Fig. 9 shows the photograph of the fabricated antenna.

TABLE I DIMENSIONS OF THE ANTENNA (UNIT: MM)

\begin{tabular}{ccccccccc}
\hline$L_{1}$ & $L_{2}$ & $L_{3}$ & $L_{4}$ & $L_{5}$ & $L_{6}$ & $L_{7}$ & $L_{8}$ & $L_{9}$ \\
\hline 198 & 10 & 8.5 & 20 & 26.6 & 1.65 & 0.95 & 2.6 & 6.5 \\
\hline \hline$L_{10}$ & $L_{11}$ & $W_{1}$ & $W_{2}$ & $W_{3}$ & $W_{4}$ & $W_{5}$ & $W_{6}$ & $W_{7}$ \\
\hline 3 & 4 & 80 & 4.6 & 8.5 & 4.2 & 7.5 & 10.5 & 18 \\
\hline \hline$W_{8}$ & $W_{9}$ & $W_{10}$ & $R_{1}$ & $R_{2}$ & $R_{3}$ & $R_{4}$ & $R_{5}$ & $R_{6}$ \\
\hline 0.4 & 0.5 & 0.5 & 5.4 & 7.1 & 3.6 & 6.4 & 0.4 & 0.7 \\
\hline
\end{tabular}

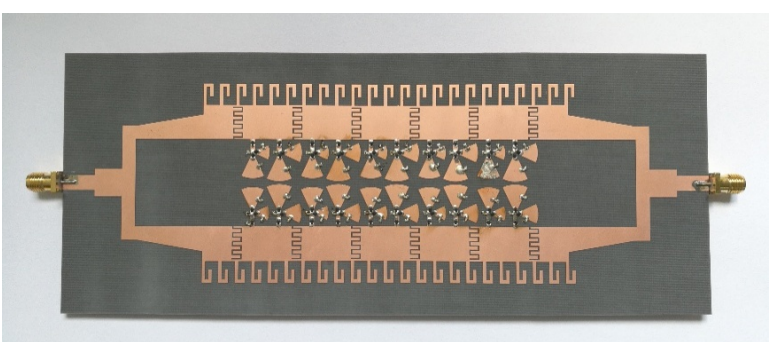

Fig. 9. Photograph of the fabricated antenna

The measured reflection coefficients for the 5 preset scanning states are plotted in Fig. 10. The measured $-10 \mathrm{~dB}$ reflection coefficient bandwidth for these switching states is $5.66-6.41 \mathrm{GHz}$. The difference between the simulation and measurement is mainly caused by the parameter errors between the real PIN diode and the equivalent circuit model used in the simulation. The manufacturing and measurement error is another reason for this difference.

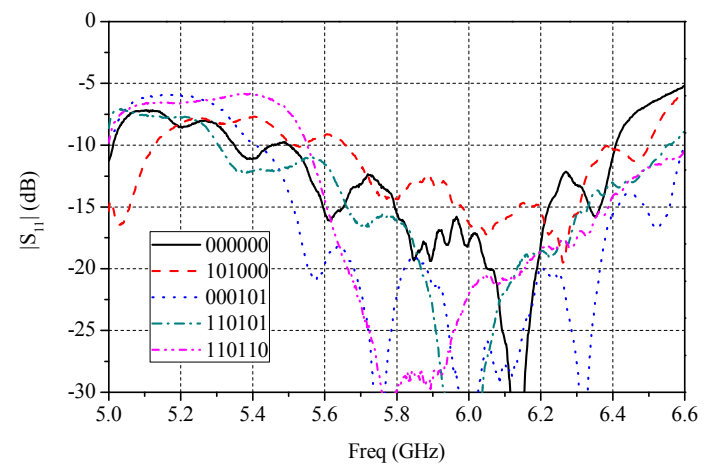

Fig. 10. Measured reflection coefficients of the proposed LWA for the 5 prese scanning states.

The measured radiation patterns of these preset scanning states are shown in Fig. 11. It is seen that the measured scan-angles of these states agree to the simulated ones. At the operation frequency of $5.8 \mathrm{GHz}$, the antenna achieves a maximum gain of $12.4 \mathrm{dBi}$. The measured gain is slightly lower than the simulated one, which is mainly caused by the parasitic parameters of the PIN diode and the manufacturing errors. The switching states corresponding to the maximum and minimum scan-angles of the antenna are "000000" and "110110", respectively. The measured scan-angle range is $34^{\circ}-59^{\circ}$ with the scanning gain variation less than $2.3 \mathrm{~dB}$.

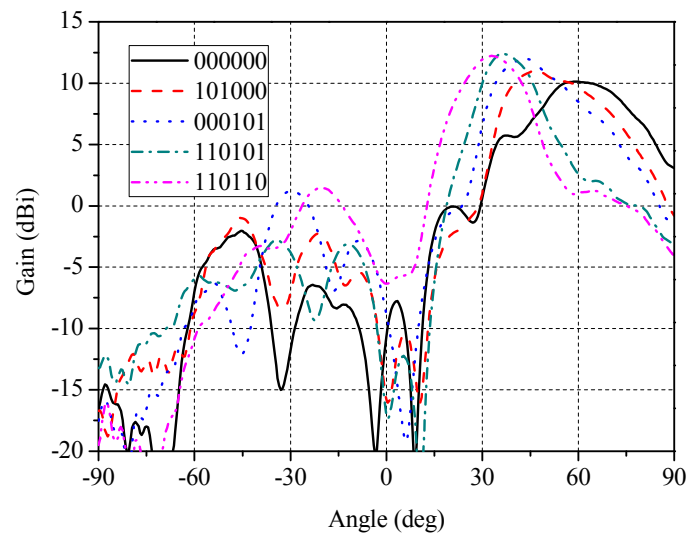

Fig. 11. Measured radiation patterns of the proposed LWA for the 5 preset scanning states at $5.8 \mathrm{GHz}$.

The scan-angle range of this LWA is limited by the phase shifting range of the electronic phase-shifting structure. In future research, the scan-angle range can be increased by using other lump components, such as varactors and inductor tunable elements.

\section{CONCLUSION}

Based on the HM-BCSIW structure, a beam scanning LWA operating at $\mathrm{C}$-band is proposed in this letter. The novel contributions from this paper lie in two points. 1) An improved CSIW structure is proposed. By using a bent open-circuit quarter-wavelength microstrip stub instead of the straight stub, the improved structure can maintain the DC-isolation between the top and bottom conductors of the SIW, while the size is reduced by about $17 \%$ compared to the original CSIW design. 2) A fixed-frequency beam scanning method for LWA is proposed. A novel electronic phase-shifting structure, which consists of fan-shaped open stubs and PIN diodes are placed between the radiating elements of the LWA for the capability of beam scanning. A prototype antenna was fabricated and measured. At the operation frequency of $5.8 \mathrm{GHz}$, the antenna achieves a measured scan-angle range of $25^{\circ}$ with the maximum gain of $12.4 \mathrm{dBi}$ and the gain variation less than $2.3 \mathrm{~dB}$. Due to the characteristics of compact structure and low profile, the proposed antenna is potentially useful for modern wireless communication systems.

\section{REFERENCES}

[1] Y. Rahmat-Samii and A. C. Densmore, "Technology Trends and Challenges of Antennas for Satellite Communication Systems," IEEE Trans. Antennas Propag., vol. 63, no. 4, pp. 1191-1204, April 2015.

[2] S. Gao, Y. Rahmat-Samii, R. E. Hodges and X. Yang, "Advanced Antennas for Small Satellites," Proc. IEEE, vol. 106, no. 3, pp. 391-403, March 2018. 
[3] Y. Li, Q. Xue, H. Tan and Y. Long, "The Half-Width Microstrip Leaky Wave Antenna With the Periodic Short Circuits," IEEE Trans. Antennas Propag., vol. 59, no. 9, pp. 3421-3423, Sept. 2011.

[4] S. A. K. Tanoli, M. I. Khan, Q. Fraz, X. Yang and S. A. Shah, "A Compact Beam-Scanning Leaky-Wave Antenna With Improved Performance," IEEE Antennas Wireless Propag. Lett., vol. 17, no. 5, pp. 825-828, May 2018.

[5] A. Pourghorban Saghati, M. M. Mirsalehi and M. H. Neshati, "A HMSIW Circularly Polarized Leaky-Wave Antenna With Backward, Broadside, and Forward Radiation," IEEE Antennas Wireless Propag. Lett., vol. 13, pp. 451-454, 2014.

[6] Y. Lyu et al., "Leaky-Wave Antennas Based on Noncutoff Substrate Integrated Waveguide Supporting Beam Scanning From Backward to Forward," IEEE Trans. Antennas Propag., vol. 64, no. 6, pp. 2155-2164, June 2016.

[7] Y. You, Y. L. Lu, G. M. Xu, Q. C. You and J. F. Huang, "Frequency-scanned antenna array based on continuous transverse stub," 2017 Progress in Electromagnetics Research Symposium, 2017, pp. 1470-1473.

[8] D. K. Karmokar, K. P. Esselle and S. G. Hay, "Fixed-Frequency Beam Steering of Microstrip Leaky-Wave Antennas Using Binary Switches," IEEE Trans. Antennas Propag., vol. 64, no. 6, pp. 2146-2154, June 2016.

[9] Y. Li, M. F. Iskander, Z. Zhang and Z. Feng, "A New Low Cost Leaky Wave Coplanar Waveguide Continuous Transverse Stub Antenna Array Using Metamaterial-Based Phase Shifters for Beam Steering," IEEE Trans. Antennas Propag., vol. 61, no. 7, pp. 3511-3518, July 2013.

[10] E. Abdo-Sánchez, D. Palacios-Campos, C. Frías-Heras, F. Y. Ng-Molina, T. M. Martín-Guerrero and C. Camacho-Peñalosa, "Electronically Steerable and Fixed-Beam Frequency-Tunable Planar Traveling-Wave Antenna," IEEE Trans. Antennas Propag., vol. 64, no. 4, pp. 1298-1306, April 2016.

[11] M. Bozzi, A. Georgiadis and K. Wu, "Review of substrate-integrated waveguide circuits and antennas," IET Microw. Antennas Propag., vol. 5 , no. 8, pp. 909-920, 6 June 2011.

[12] Y. Dong and T. Itoh, "Composite Right/Left-Handed Substrate Integrated Waveguide and Half Mode Substrate Integrated Waveguide Leaky-Wave Structures," IEEE Trans. Antennas Propag., vol. 59, no. 3, pp. 767-775, March 2011.

[13] W. Cao, Z. N. Chen, W. Hong, B. Zhang and A. Liu, "A Beam Scanning Leaky-Wave Slot Antenna With Enhanced Scanning Angle Range and Flat Gain Characteristic Using Composite Phase-Shifting Transmission Line," IEEE Trans. Antennas Propag., vol. 62, no. 11, pp. 5871-5875, Nov. 2014

[14] S. S. Haghighi, A. Heidari and M. Movahhedi, "A Three-Band Substrate Integrated Waveguide Leaky-Wave Antenna Based on Composite Right/Left-Handed Structure," IEEE Trans. Antennas Propag., vol. 63, no. 10 , pp. 4578-4582, Oct. 2015.

[15] D. G. Chen and K. W. Eccleston, "Substrate integrated waveguide with corrugated wall," 2008 Asia-Pacific Microwave Conference, 2008, pp. $1-4$.

[16] K. W. Eccleston, "Corrugated substrate integrated waveguide distributed amplifier," 2012 Asia Pacific Microwave Conference, 2012, pp. 379-381. 